\title{
Thermal conductivity of compound semiconductors: Interplay of mass density and acoustic-optical phonon frequency gap
}

\author{
Ankit Jain and Alan J. H. McGaughey ${ }^{a)}$ \\ Department of Mechanical Engineering, Carnegie Mellon University, Pittsburgh, Pennsylvania 15213, USA
}

(Received 23 May 2014; accepted 4 August 2014; published online 18 August 2014)

\begin{abstract}
The thermal conductivities of model compound semiconductors, where the two species differ only in mass, are predicted using lattice dynamics calculations and the Boltzmann transport equation. The thermal conductivity varies non-monotonically with mass ratio, with a maximum value that is four times higher than that of a monatomic semiconductor of the same density. The very high thermal conductivities are attributed to a reduction in the scattering of optical phonons when the acoustic-optical frequency gap in the phonon dispersion approaches the maximum acoustic phonon frequency. The model system predictions compare well to predictions for real compound semiconductors under appropriate scaling, suggesting a universal behavior and a strategy for efficient screening of materials for high thermal conductivity. (C) 2014 AIP Publishing LLC.
\end{abstract}

[http://dx.doi.org/10.1063/1.4893185]

\section{INTRODUCTION}

Thermal transport in crystalline semiconductors is primarily due to atomic vibrations that are quantized as phonons. ${ }^{1}$ The parameters that affect phonon transport include the lattice structure, the atomic masses, anharmonicity, and defects (e.g., isotopes, dopants, dislocations, and interfaces). ${ }^{1-7}$ For application in thermoelectric energy conversion, many previous studies have focused on how to reduce thermal conductivity. ${ }^{8-10}$ Phonon scattering increases with increasing anharmonicity and defect concentration, resulting in lower thermal conductivity. In single species (i.e., monatomic) materials, larger atomic mass reduces the phonon group velocities and, thus, thermal conductivity.

To identify the features of high thermal conductivity semiconductors, Slack studied diamond, boron nitride, boron phosphide, silicon carbide, and aluminum nitride. ${ }^{11} \mathrm{He}$ concluded that high thermal conductivity is correlated to strong atomic interactions, low anharmonicity, simple crystal structure, and low average atomic mass. Based on these criteria, he predicted a monotonic variation of thermal conductivity with mass density and estimated the room temperature thermal conductivity of boron arsenide (BAs) to be $210 \mathrm{~W} / \mathrm{m}-\mathrm{K}$. Recently, Lindsay et al. ${ }^{12}$ and Broido et al. ${ }^{13}$ studied phonon transport in cubic boron compound semiconductors using first principles calculations and found a non-monotonic thermal conductivity variation with mass density. At a temperature of $300 \mathrm{~K}$, their highest predicted thermal conductivity of $3170 \mathrm{~W} / \mathrm{m}-\mathrm{K}$ was for isotopically pure BAs, which compares to that of isotopically pure diamond. ${ }^{14,15}$ They attributed the ultrahigh thermal conductivity of BAs to a large acousticoptical (a-o) frequency gap in the phonon dispersion, acoustic bunching, strong covalent bonding, and low anharmonicity. The relative importance of each of these effects in determining the thermal conductivity, however, is unknown. A non-monotonic variation of thermal conductivity with

${ }^{\text {a)} E l e c t r o n i c ~ a d d r e s s: ~ m c g a u g h e y @ c m u . e d u ~}$ mass ratio was also observed by Steigmeier and Kudman for a subset of group III-V semiconductors based on experimental measurements. ${ }^{16}$

Our objective, in this work, is to study the competing effects of the mass density and the phonon dispersion (i.e., a-o gap, acoustic bunching, and acoustic and optical widths) on the thermal conductivity of compound semiconductors. The rest of the paper is organized as follows. In Sec. II, the model system is presented and the lattice dynamics- and Boltzmann transport equation (BTE)-based methodology for predicting phonon properties and thermal conductivity is described. The thermal conductivity predictions are presented and interpreted in Sec. III, where we argue that an a-o gap that is comparable to or larger than the maximum acoustic phonon frequency is required to obtain high thermal conductivities in compound semiconductors. The model system predictions are compared to available data for a range of compound semiconductors in Sec. IV.

\section{METHODOLOGY}

\section{A. Lattice dynamics calculations}

We use a defect-free zinc-blende lattice where the mass of one atom in the primitive unit cell $\left(m_{1}\right)$ is fixed at that of silicon, while the second atom's mass $\left(m_{2}\right)$ is varied from $0.1 m_{1}$ to $10 m_{1}$. The mass ratio is defined as $m_{2} / m_{1}$ and values of $0.1,0.2,0.25,0.3,0.4,0.5,0.6,0.7,0.8,0.9,1,2,3,4,5$, $6,7,8,9$, and 10 are considered. To isolate the effects of the phonon dispersion and mass density on thermal conductivity, we use classical (i.e., Boltzmann) statistics at a temperature of $500 \mathrm{~K}$. Doing so compared to quantum statistics removes the frequency dependence of the specific heat from the thermal conductivity prediction. The lattice positions in the zincblende structure are equivalent. For classical statistics, the thermal conductivity for a system with mass ratio of $m_{2} / m_{1}$ is proportional to that of a system with ratio $m_{1} / m_{2}$ through the scaling factor of $\left[\left(m_{1}+m_{2}\right) /\left(m_{1}+1 / m_{2}\right)\right]^{-1 / 2}$. ${ }^{17}$ The 
Stillinger-Weber potential as parameterized for silicon ${ }^{18}$ is used to model all atomic interactions so that we can focus on the effects of the mass density and the dispersion.

The phonon contribution to the thermal conductivity of a bulk crystalline semiconductor in the $l$ direction can be calculated by solving the BTE and using the Fourier law and is ${ }^{19,20}$

$$
k_{l}=\sum_{i} c_{p h, i} v_{g, l, i}^{2} \tau_{l, i}
$$

The summation in Eq. (1) is over all the phonon modes in the first Brillouin zone. The mode index, $i \equiv\left(\begin{array}{l}\boldsymbol{\kappa} \\ \nu\end{array}\right)$, enumerates phonon wave vector, $\boldsymbol{\kappa}$, and polarization, $\nu$. On the right-hand side of Eq. (1), $c_{p h, i}$ is the volumetric specific heat, $v_{g, l, i}$ is the $l$-component of the phonon group velocity vector $\mathbf{v}_{g, i}$, and $\tau_{l, i}$ is the phonon lifetime. The specific heat in classical statistics is $k_{\mathrm{B}} / V$, where $k_{\mathrm{B}}$ is the Boltzmann constant and $V$ is the system volume. The group velocity vector is related to the mode frequency, $\omega_{i} \equiv \omega_{\nu}^{\boldsymbol{\kappa}}$, as $\mathbf{v}_{g, i}=\partial \omega_{\nu}^{\boldsymbol{\kappa}} / \partial \boldsymbol{\kappa}$. Under the commonly used relaxation time approximation (RTA) of the BTE, the phonon lifetime can be estimated using the inverse of the phonon scattering rate, $\frac{\partial n_{i}}{\partial t} \cdot{ }^{19,21,22}$

Harmonic lattice dynamics calculations are used to obtain the phonon frequencies. ${ }^{23}$ The dynamical matrix, $\mathbf{D}(\boldsymbol{\kappa})$, has elements

$$
D_{\tau \tau^{\prime}}^{\alpha \alpha^{\prime}}(\boldsymbol{\kappa})=\sum_{\mathbf{R}^{\prime}} \frac{1}{\sqrt{m_{\tau} m_{\tau^{\prime}}}} \Phi_{\mathbf{0} \tau, \mathbf{R}^{\prime} \tau^{\prime}}^{\alpha \alpha^{\prime}} \exp \left(\mathrm{i} \boldsymbol{\kappa} \cdot \mathbf{R}^{\prime}\right) .
$$

Here, the summation is over all unit cells, which are located at position vector $\mathbf{R}^{\prime}$, and $m_{\tau}$ is the mass of atom $\tau$ in the unit cell. $\Phi_{\mathbf{0} \tau, \mathbf{R}^{\prime} \tau^{\prime}}^{\alpha \alpha^{\prime}}$ is the second-order (i.e., harmonic) force constant linking the motion of atom $(\mathbf{0}, \tau)$ (atom $\tau$ in the unit cell at $\mathbf{0})$ in the $\alpha$-direction $(\alpha=x, y$, or $z)$ and atom $\left(\mathbf{R}^{\prime}, \tau^{\prime}\right)$ in the $\alpha^{\prime}$-direction. The phonon frequencies and eigenvectors, $\mathbf{e}_{\nu}^{\boldsymbol{\kappa}}$, can then be obtained by solving the eigenvalue problem

$$
\left(\omega_{\nu}^{\boldsymbol{\kappa}}\right)^{2} \mathbf{e}_{\nu}^{\boldsymbol{\kappa}}=\mathbf{D}(\boldsymbol{\kappa}) \mathbf{e}_{\nu}^{\boldsymbol{\kappa}} .
$$

We consider three-phonon anharmonic processes under the RTA. The phonon scattering rates are calculated from ${ }^{19,22}$

$$
\begin{aligned}
\frac{\partial n_{i}}{\partial t} \equiv \frac{\partial n_{\nu}^{\boldsymbol{\kappa}}}{\partial t}= & \frac{\pi \hbar}{16 N} \sum_{\boldsymbol{\kappa}^{\prime} \nu^{\prime}} \sum_{\boldsymbol{\kappa}^{\prime \prime} \nu^{\prime \prime}}\left|V_{\nu \nu^{\prime} \nu^{\prime \prime}}^{\boldsymbol{\kappa} \boldsymbol{\kappa}^{\prime} \boldsymbol{\kappa}^{\prime \prime}}\right|^{2}\left\{\left(f_{\nu^{\prime}}^{\boldsymbol{\kappa}^{\prime}}+f_{\nu^{\prime \prime}}^{\boldsymbol{\kappa}^{\prime \prime}}+1\right) \delta\left(\omega_{\nu}^{\boldsymbol{\kappa}}-\omega_{\nu^{\prime}}^{\boldsymbol{\kappa}^{\prime}}-\omega_{\nu^{\prime \prime}}^{\boldsymbol{\kappa}^{\prime \prime}}\right)\right. \\
& \left.+\left(f_{\nu^{\prime}}^{\boldsymbol{\kappa}^{\prime}}-f_{\nu^{\prime \prime}}^{\boldsymbol{\kappa}^{\prime \prime}}\right)\left[\delta\left(\omega_{\nu}^{\boldsymbol{\kappa}}+\omega_{\nu^{\prime}}^{\boldsymbol{\kappa}^{\prime}}-\omega_{\nu^{\prime \prime}}^{\boldsymbol{\kappa}^{\prime \prime}}\right)-\delta\left(\omega_{\nu}^{\boldsymbol{\kappa}}-\omega_{\nu^{\prime}}^{\boldsymbol{\kappa}^{\prime}}+\omega_{\nu^{\prime \prime}}^{\boldsymbol{\kappa}^{\prime \prime}}\right)\right]\right\}
\end{aligned}
$$

The summations in Eq. (4) are over all the phonon modes, $\hbar$ is the reduced Planck constant, $N$ is the total number of phonon modes, $f_{\nu}^{\boldsymbol{\kappa}}$ is the equilibrium phonon occupation number, and $\delta()$ is the delta function. $V_{\nu \nu^{\prime} \nu^{\prime \prime}}^{\boldsymbol{\kappa} \boldsymbol{\kappa}^{\prime \prime}}$ is the three-phonon scattering matrix element, which depends on the third-order force constants $\Phi_{0 \tau, \mathbf{R}^{\prime} \tau^{\prime \prime}, \mathbf{R}^{\prime \prime} \tau^{\prime \prime}}^{\alpha \prime \prime}$ and is defined as

$$
\begin{aligned}
V_{\nu \nu^{\prime} \nu^{\prime \prime}}^{\boldsymbol{\kappa} \boldsymbol{\kappa}^{\prime}}= & \sum_{\mathbf{R}^{\prime \prime} \tau^{\prime \prime} \alpha^{\prime \prime}} \sum_{\mathbf{R}^{\prime} \tau^{\prime} \alpha^{\prime}} \sum_{\tau \alpha} \delta\left(\boldsymbol{\kappa}+\boldsymbol{\kappa}^{\prime}+\boldsymbol{\kappa}^{\prime \prime}-\mathbf{G}\right) \\
& \times \Phi_{0 \tau, \mathbf{R}^{\prime} \tau^{\prime \prime}, \mathbf{R}^{\prime \prime} \tau^{\prime \prime}}^{\alpha \alpha^{\prime} \alpha^{\prime \prime}} \times \frac{e^{\mathrm{i}\left(\boldsymbol{\kappa}^{\prime} \cdot \mathbf{R}^{\prime}+\boldsymbol{\kappa}^{\prime \prime} \cdot \mathbf{R}^{\prime \prime}\right)} e_{\nu \tau}^{\boldsymbol{\kappa} \alpha} e_{\nu^{\prime} \tau^{\prime}}^{\boldsymbol{\kappa}^{\prime} \alpha_{\nu^{\prime}}^{\prime \prime} \tau^{\prime \prime}}}{\sqrt{m_{\tau} m_{\tau^{\prime}} m_{\tau^{\prime \prime}} \omega_{\nu}^{\boldsymbol{\kappa}} \omega_{\nu^{\prime}}^{\boldsymbol{\kappa}^{\prime}} \omega_{\nu^{\prime \prime}}^{\boldsymbol{\kappa}^{\prime \prime}}}},
\end{aligned}
$$

where $\mathbf{G}$ is a reciprocal lattice vector and $e_{\nu \tau}^{\boldsymbol{\kappa} \alpha}$ is the $\alpha$-component of the eigenvector $\mathbf{e}_{\nu}^{\boldsymbol{\kappa}}$ for atom $\tau$.

Phonon-phonon scattering processes must satisfy the delta functions (i.e., the selection rules) in Eq. (4) (energy conservation) and Eq. (5) (lattice translational invariance). ${ }^{1}$ In compound semiconductors, for acoustic (a) phonons, a-a-a, a-a-o, and a-o-o processes are generally possible, where "o" denotes an optical phonon. For optical phonons, because of their high frequencies and narrow width, o-o-o processes typically cannot satisfy energy conservation. Optical phonons, therefore, generally only scatter through a-o-o and a-a-o processes (i.e., they require an acoustic phonon). ${ }^{12}$ We note that all phonon modes are active in the classical systems we are studying. As such, all phonon-phonon scattering processes that satisfy the selection rules can occur.

\section{B. Phonon wave vector grid density dependence of thermal conductivity}

We predict thermal conductivity using a phonon wavevector grid with an equal number of uniformly-spaced grid points, $N_{o}$, in each direction (i.e., $N=N_{o}^{3}$ ). Of the systems considered here, we find the maximum thermal conductivity at a mass ratio of $0.25 .^{24}$ The thermal conductivity for this system is plotted in Fig. 1(a) vs $N_{o}$. The green squares, purple crosses, and orange circles represent the acoustic phonon contribution, the optical phonon contribution, and the total thermal conductivity. The acoustic phonon contribution converges after $N_{o}=32$. Because of the low scattering rate of optical phonons at this mass ratio (to be discussed in Sec. III), their contribution continues to decrease even at $N_{o}=64$. To resolve this issue, we obtain the bulk thermal conductivity by plotting $1 / k$ (the inverse of the total thermal conductivity) vs. $1 / N_{o}$ in Fig. 1(b) and extrapolating a linear fit to the $N_{o} \rightarrow \infty$ limit. Systems with $N_{o}=(24,28,32,36,40,44)$ [open circles in Fig. 1(b)] are used for fitting and extrapolation. The solid circles $\left[N_{o}=(48,52,56,60,64)\right]$ are used to assess the quality of the fit. The extrapolation technique works well and all non-fitted points lie on the fitted line. We note that $1 / k$ increases with decreasing $1 / N_{o}$. This behavior is opposite to that observed in other studies, where $1 / k$ decreases with decreasing $1 / N_{o} \cdot{ }^{21,25}$ In those cases, the $1 / k$ vs $1 / N_{o}$ fitting and extrapolation is used to include the contribution of acoustic phonon modes close to the center of the 
Brillouin zone. We observe this effect in our systems typically for $N_{o}<22$ and those data are not used for fitting.

We employ $N_{o}=44$ to specify the acoustic phonon thermal conductivity for all mass ratios. For cases where the total thermal conductivity is not converged at $N_{o}=44$ (mass ratios of 0.1 to 0.3 and 4 to 10$), N_{o}=(24,28,32,36,40,44)$ systems are used to perform the $1 / k$ vs $1 / N_{o}$ fitting and extrapolation. The optical contribution is then obtained by subtracting the acoustic phonon contribution at $N_{o}=44$ from the extrapolated total thermal conductivity.

\section{RESULTS}

\section{A. Dispersion}

We first consider the phonon dispersion to study the effect of mass ratio on the a-o gap, bunching, and acoustic and optical widths. For the analysis to follow, we define: (i) the a-o gap as the difference between the maximum acoustic frequency and the minimum optical frequency, (ii) the acoustic (optical) width as the difference between the maximum and minimum acoustic (optical) frequencies, and (iii) the acoustic bunching as the maximum difference between the maximum frequencies of the three acoustic phonon

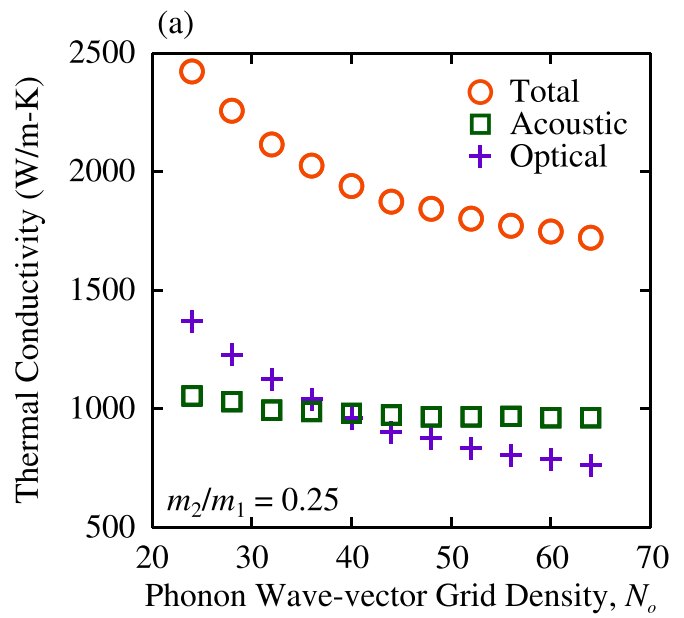

(b)

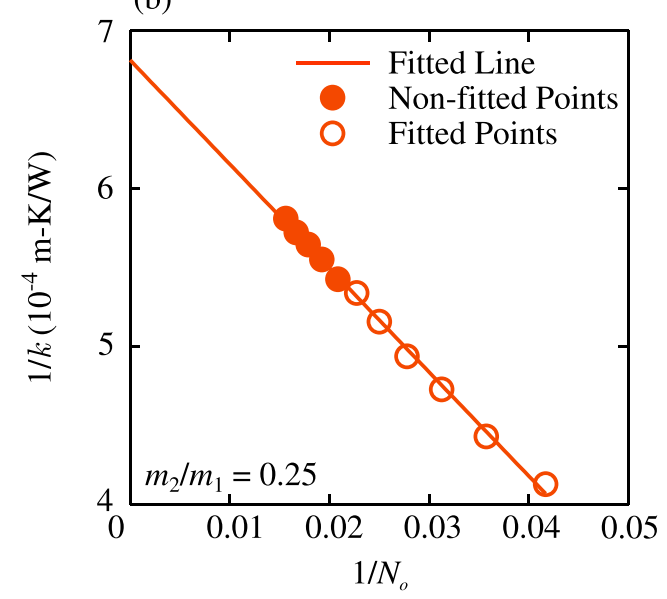

FIG. 1. For the system with a mass ratio of 0.25: (a) Variation of total, acoustic, and optical thermal conductivities with phonon wave-vector grid density. The acoustic contribution converges after $N_{o}=32$. (b) $1 / k$ versus $1 / N_{o}$ and linear fit for extrapolation to the bulk thermal conductivity. The line is only fitted to the six rightmost points (open circles). (a)

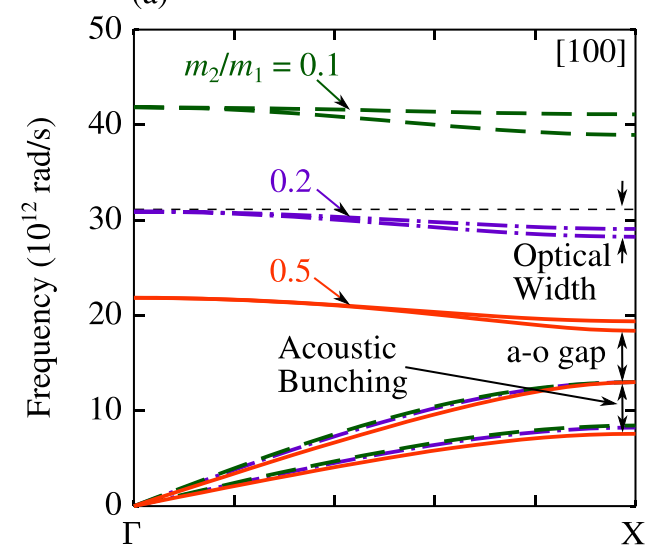

(b)

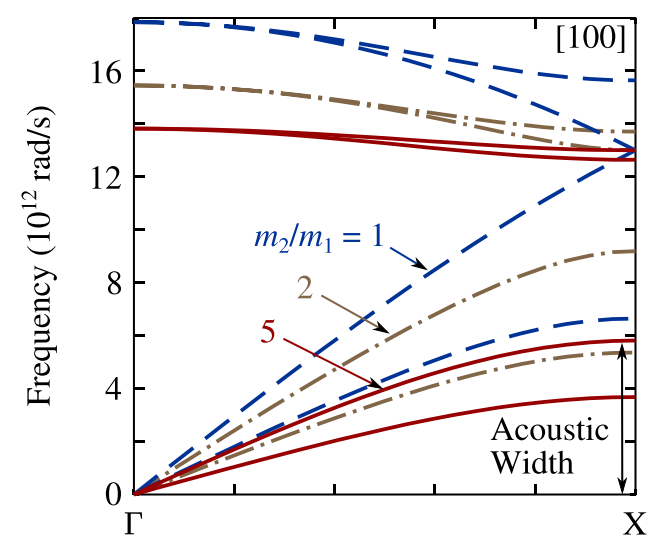

FIG. 2. Effect of mass ratio on the [100] phonon dispersion for (a) $m_{2} / m_{1}$ $<1$ and (b) $m_{2} / m_{1} \geq 1$. The a-o gap, the acoustic bunching, the acoustic width, and the optical width are identified for select cases. In the analysis, these quantities are calculated based on the entire first Brillouin zone.

polarizations. ${ }^{12}$ The maximum and minimum frequencies in these definitions are based on the entire first Brillouin zone.

In Fig. 2(a), the dispersion in the [100] direction is plotted for mass ratios less than 1 . The optical phonon frequencies increase with a reduction in the mass ratio, while the acoustic phonons are largely unaffected. As a result, the a-o gap increases, while the acoustic width and bunching remain unchanged as mass ratio is reduced. The effect of increasing the mass ratio beyond 1 is shown in Fig. 2(b) for the [100] direction. The a-o gap increases and the acoustic width and bunching decrease with an increase in the mass ratio. For the monatomic system (mass ratio $=1$ ), the acoustic and optical branches intersect at the zone boundary (i.e., the a-o gap is zero). The optical width is a maximum for the monatomic system and decreases with increasing and decreasing mass ratios.

\section{B. Thermal conductivity}

The total and acoustic thermal conductivities are plotted in Fig. 3(a) versus the mass ratio. Also plotted is the total thermal conductivity of a monatomic system with the same mass density as the corresponding compound semiconductor. For the monatomic system, the thermal conductivity scales as $\left(m_{1}+m_{2}\right)^{-1 / 2}$ and is always lower than that of the corresponding compound semiconductor. 


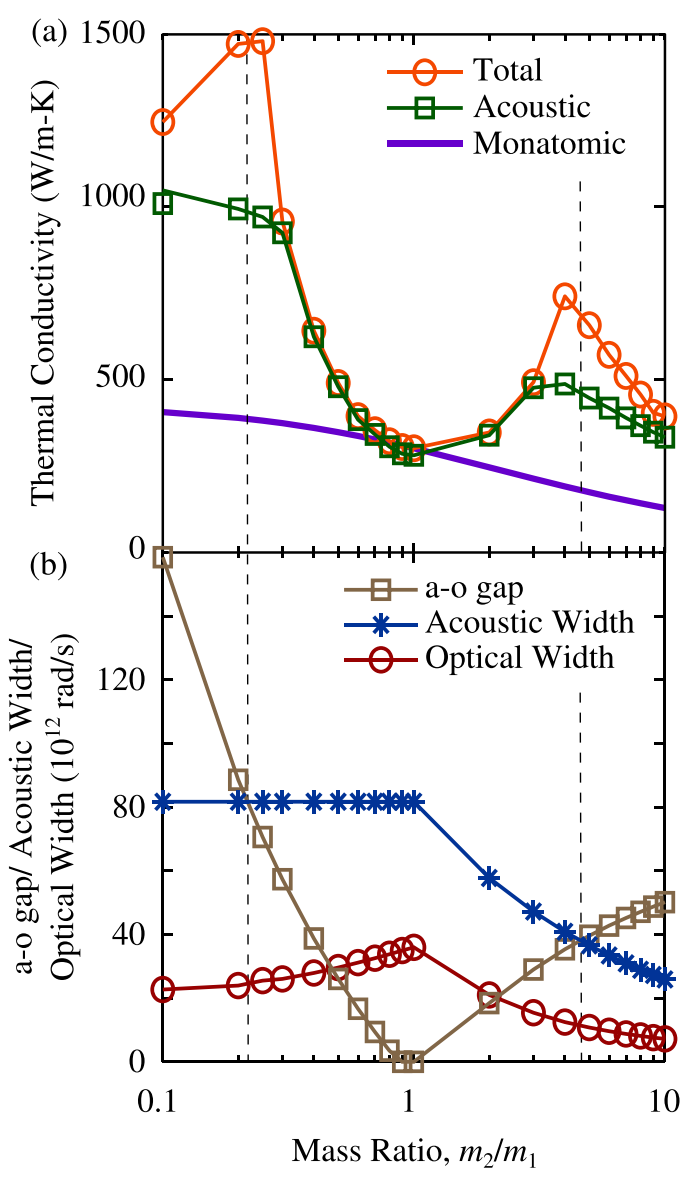

FIG. 3. (a) Total and acoustic thermal conductivity variation with mass ratio, and (b) a-o gap, acoustic width, and optical width variation with mass ratio. In (a), the purple line corresponds to the thermal conductivity of a monatomic semiconductor that has the same mass density as the corresponding compound semiconductor. The dashed vertical lines represent where the a-o gap and the acoustic width are equal.

For the compound semiconductors, the minimum total thermal conductivity occurs at a mass ratio of 1 , where it equals that of the monatomic system. Moving away from the mass ratio of 1 , the total thermal conductivity initially increases with increasing mass ratio, reaches a peak at a mass ratio of 4 , then decreases with further increase in the mass ratio. As expected, due to the mass ratio scaling, the trend is similar for mass ratios smaller than 1 , where the peak in total thermal conductivity occurs at a mass ratio of 0.25 . This behavior of total thermal conductivity in compound semiconductors is in contrast to that in monatomic semiconductors, where thermal conductivity monotonically decreases with increasing mass ratio.

The acoustic thermal conductivity is $93 \%$ of the total thermal conductivity at a mass ratio of 1 . On increasing the mass ratio, the acoustic thermal conductivity follows the total thermal conductivity until the mass ratio reaches 3 . The contribution of optical phonons to the total thermal conductivity increases from $4 \%$ for a mass ratio of 3 to $34 \%$ for mass ratio of 4 . The acoustic thermal conductivity thus deviates from the total thermal conductivity beyond a mass ratio of 4, decreasing with further increase in the mass ratio. Between mass ratios of 4 and 10, the acoustic thermal conductivity decreases from 487 to $333 \mathrm{~W} / \mathrm{m}-\mathrm{K}$ (i.e., by $32 \%$ ) which is comparable to the decrease in the thermal conductivity of the monatomic semiconductor in this regime $(33 \%)$. This decrease in thermal conductivity is mainly due to the reduction in phonon group velocities with an increase in the mass density. For mass ratios less than 1, the acoustic thermal conductivity increases with decreasing mass ratio, but deviates from the total thermal conductivity for mass ratios below 0.4 , where the optical phonon contribution strongly increases.

\section{Role of a-o gap}

To understand the origin of the peaks in the total thermal conductivity and the large contribution of optical phonons for certain mass ratios, we plot the variation of the a-o gap, the acoustic width, and the optical width with mass ratio in Fig. 3(b). The locations of the crossings of the a-o gap and the acoustic-width curves are shown in Fig. 3 using vertical dashed lines.

In the neighborhood of a mass ratio of 1 , the contribution of optical phonons to the total thermal conductivity of the compound semiconductor is $7 \%$. With increasing mass ratio, the a-o gap widens, resulting in fewer a-a-o processes. $^{26}$ The acoustic and optical thermal conductivities therefore both increase with increasing mass ratio in this region. At a mass ratio of 4 , the a-o gap is $86 \%$ of the acoustic-width, thereby reducing a-a-o processes significantly. The optical phonon contribution to thermal conductivity increases to $34 \%$. Soon after this point, a-a-o processes are eliminated and optical phonons can only scatter through a-o-o processes, while acoustic phonons can still scatter through a-a-a and a-o-o processes. For mass ratios greater than 4 , the mass density effect (reduction in phonon group velocities with an increase in the mass density) dominates the thermal conductivity trend and the acoustic and optical contributions decrease with increasing mass ratio.

With a reduction in the mass ratio below 1 , both the mass density and a-o gap effects favor an increase in the thermal conductivity. The acoustic thermal conductivity, therefore, increases with decreasing mass ratio. For optical phonons, the thermal conductivity increases with decreasing mass ratio up to a mass ratio of 0.25 and then decreases with further decrease in the mass ratio. In Sec. III D, we will argue that this decrease is a result of a reduction in the already small group velocities of optical phonons.

\section{Roles of acoustic bunching, acoustic width, and optical width}

To this point, we have not considered the effects of acoustic bunching, acoustic width, and optical width. Bunching affects the phonon-phonon scattering rates ${ }^{12,13}$ and the widths affect the group velocities. The effect of these changes on thermal conductivity is now analyzed.

In Figs. 4(a) and 4(b), the mass-ratio-dependent thermal conductivities, a-o gaps, acoustic widths, and optical widths from Figs. 3(a) to 3(b) are replotted after scaling with $\left(m_{1}+m_{2}\right)^{1 / 2}$. This scaling factor eliminates any dependence of thermal conductivity on the mass density of the system. 


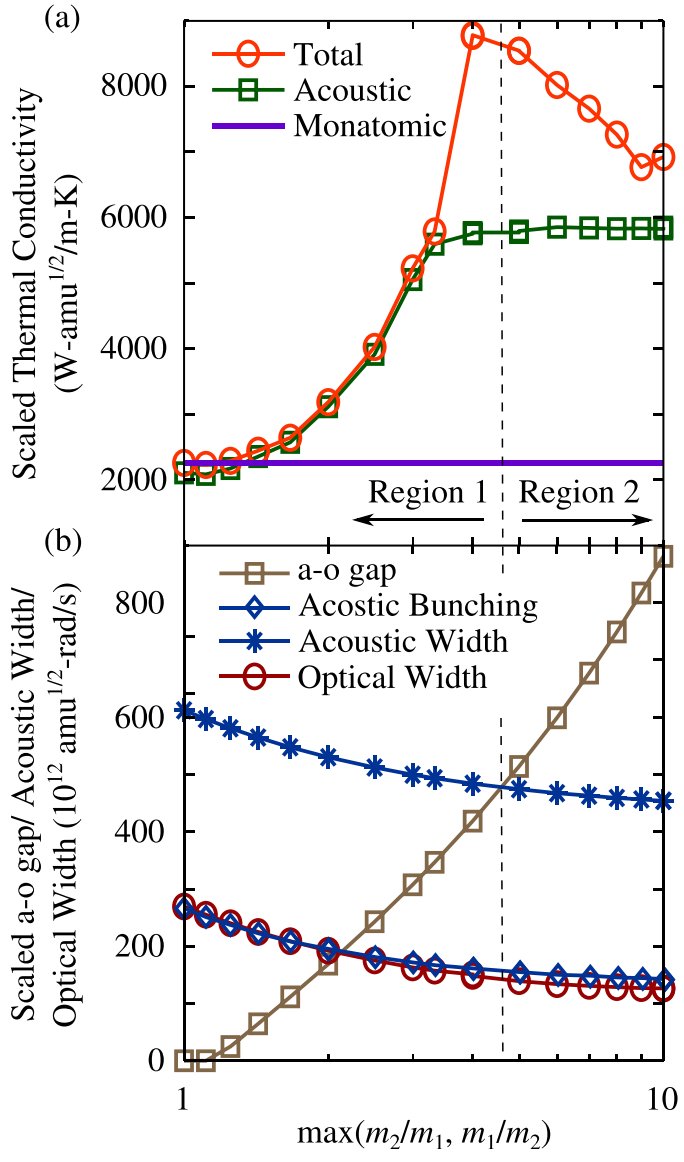

FIG. 4. (a) Scaled total and acoustic thermal conductivity variation with mass ratio, and (b) scaled a-o gap, acoustic bunching, acoustic width, and optical width variation with mass ratio. The scaling factor of $\left(m_{1}+m_{2}\right)^{1 / 2}$ is used in order to eliminate the effect of mass density.

Also plotted in Fig. 4(b) is the acoustic bunching. The data are plotted versus the maximum of $m_{2} / m_{1}$ and $m_{1} / m_{2}$ and, as expected, collapse onto one curve (i.e., the scaled value for a mass ratio of $m_{2} / m_{1}$ is the same as that for $\left.m_{1} / m_{2}\right)$. Also, as expected, the scaled thermal conductivity of the monatomic system is constant with mass ratio. The location of the crossing of the a-o gap and the acoustic-width curves is shown in Fig. 4 using a vertical dashed line. The region to the left of the line has a-o gaps less than the acoustic width (Region 1) and region to the right has a-o gaps greater than the acoustic width (Region 2). In Region 2, a-a-o processes cannot satisfy the energy conservation selection rule. The a-o gap therefore has minimal effect on either acoustic or optical thermal conductivities in this region. The acoustic and optical thermal conductivities in Region 2 are, therefore, mainly affected by acoustic bunching, acoustic width, and optical width. We note that the peak total thermal conductivity occurs just before the a-o gap exceeds the acoustic width.

The scaled acoustic bunching and scaled acoustic width in Region 2 decreases by $8 \%$ and $4 \%$ between mass ratios of 5 and 10 , while the scaled acoustic thermal conductivity is almost constant (decreases by $1 \%$ ). This result suggests that there is a minimal effect of acoustic bunching and acoustic width on the acoustic thermal conductivity for this model system. The scaled optical thermal conductivity in Region 2 decreases by $61 \%$ for a $9 \%$ decrease in the optical width.
This result suggests that the reduction in the optical phonon group velocities dominates over an increase in their lifetimes due to the reduced scattering.

\section{COMPARISON WITH REAL COMPOUND SEMICONDUCTORS}

Our results suggest that thermal conductivity can increase or decrease with a change in mass density depending on the relative sizes of the a-o gap and the acoustic width. For materials that have an a-o gap less than the acoustic-width, the total, acoustic, and optical thermal conductivity trends are predominantly decided by the a-o gap, with thermal conductivity increasing with increasing a-o gap. For materials that have an a-o gap greater than the acoustic-width, the acoustic and the optical thermal conductivities decrease with increasing mass density. Recently, Mukhopadhyay and Stewart also observed an a-o gap dependent thermal conductivity for cubic $\mathrm{BN}$ through the application of mechanical stress. ${ }^{27}$

In our model system, we kept the same atomic interactions for all mass ratios. To check the applicability of our findings to real materials, we now compare our model system predictions to available data for a range of monatomic and compound semiconductors. The materials considered have a zinc-blende structure and are from group III-V (BN, BP, BAs, BSb, AlP, AlAs, AlSb, GaP, GaAs, GaSb, InP, InAs, and $\mathrm{InSb}$ ), group II-VI (BeS, BeSe, and BeTe), and group IV ( $\mathrm{Si}$, $\mathrm{Ge}, \mathrm{SiC}, \mathrm{GeC}$, and diamond). The thermal conductivities used are from first-principles calculations of defect-free isotopically pure structures at a temperature of $300 \mathrm{~K}$, as reported by Lindsay et al. ${ }^{12,20,28}$ We use these first-principles based thermal conductivities predictions because experimental measurements are not available for isotopically pure compounds.

To compare the data, all thermal conductivities are scaled by the Slack parameter

$$
1 /\left(\bar{m} a \theta_{\mathrm{D}}^{3} / T\right)
$$

where $\bar{m}$ is the average atomic mass in the unit cell, $a$ is the lattice constant, $\theta_{\mathrm{D}}$ is the Debye temperature, ${ }^{29}$ and $T$ is the temperature. ${ }^{11,30}$ The Slack parameter attempts to normalize the effects of mass density, crystal structure, atomic interactions, and temperature on the thermal conductivity. Before scaling the thermal conductivities of the model system, the values are first normalized so that the $m_{2} / m_{1}=1$ system has the same thermal conductivity as first-principles silicon at a temperature of $500 \mathrm{~K}$. Based on the findings in Sec. III, the thermal conductivities are plotted in Fig. 5 versus the ratio of the a-o gap to the acoustic width. The phonon dispersions needed to specify the a-o gap and the acoustic width for the real materials are calculated using density functional perturbation theory as implemented in the plane-wave based density functional theory package Quantum Espresso. ${ }^{31,32}$

The scaled thermal conductivity of the model system increases with increasing a-o gap/acoustic width ratio, reaches a peak when the ratio is 0.86 , and then decreases with further increase in the ratio. The trend is similar for the compound semiconductors, where the peak in scaled thermal 


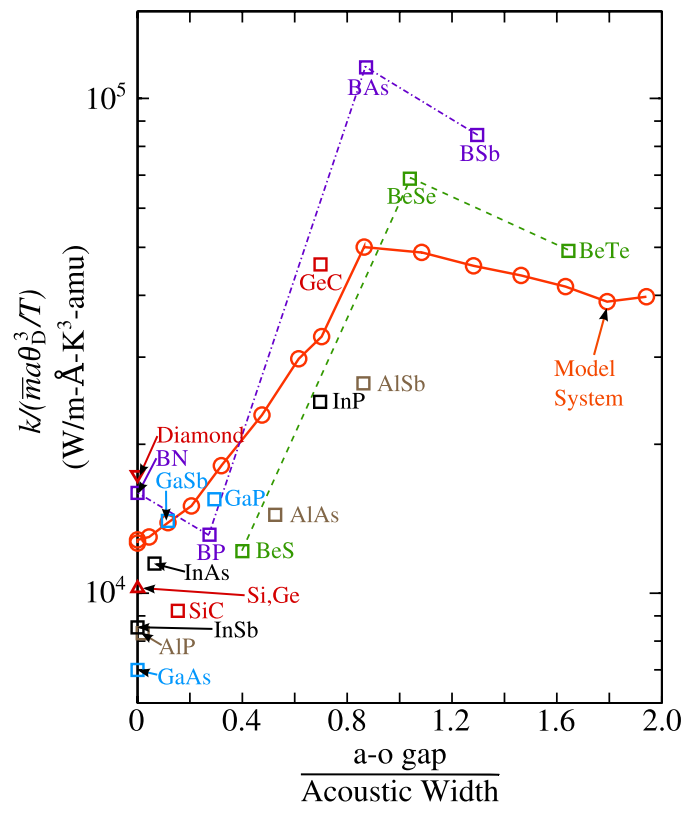

FIG. 5. Thermal conductivity variation of real compound semiconductor (squares), monatomic semiconductor (triangles), and our model system (circles) versus the a-o gap to acoustic-width ratio. The thermal conductivities are scaled by $1 /\left(\bar{m} a \theta_{\mathrm{D}}^{3} / T\right)$. Dashed lines are added to the $\mathrm{BX}$ and $\mathrm{BeX}$ series to guide the eye.

conductivity is for BAs, which has an a-o gap to acousticwidth ratio of 0.87 . As observed by Lindsay et al., ${ }^{12}$ the thermal conductivity varies non-monotonically for BX compounds (purple points). The same is true for BeX compounds (green), where the scaled thermal conductivity is a maximum for BeSe, which has an a-o gap to acoustic-width ratio of 1.03. All other compounds [AlX (brown), GaX (blue), InX (black)] have an a-o gap less than the acoustic width. As a result, thermal conductivity increases with increasing ratio and there is no peak in the scaled thermal conductivities of these materials. We note that for the BX compounds, thermal conductivity decreases in going from BN to BP. We believe that the deviations between the real materials and our model system predictions are due to the lack of anharmonicity in the scaling factor and the RTA solution of the BTE. The overall agreement in the trend, however, suggests that we have identified a universal behavior.

\section{SUMMARY}

We used lattice dynamics calculations and the BTE to study the competing effects of mass density, a-o gap, acoustic width, optical width, and acoustic bunching on the thermal conductivity of a family of model compound semiconductors, where the species differ only in mass. As shown in Figs. 3 and 4, we found that thermal conductivity depends strongly on the mass difference of the two species and how it affects the a-o gap and the acoustic-width. The thermal conductivity increases with increasing a-o gap to acoustic-width ratio, attains a maximum close to ratio of 1 , and decreases with further increase in the ratio. The observed peak in the thermal conductivity is due to the competing effects of mass density (phonon group velocities, affected by widths) and phonon-phonon scattering rates (mainly affected by a-o gap to acoustic-width ratio).

Our findings hold true for real compound semiconductors, where the atomic interactions also vary with the atomic species. As shown in Fig. 5, the scaling factor proposed by Slack along with the a-o gap to acoustic-width ratio can be used to estimate the thermal conductivity of a wide variety of compound semiconductors. The material properties involved in the Slack scaling factor and the a-o gap to acoustic-width ratio are all based on the material structure and phonon dispersion and provide a simple measure for future screening of high thermal conductivity materials.

We arrived at the results in this study by considering only three-phonon scattering processes, classical statistics for phonons at one temperature, and the RTA solution of the BTE. $^{24}$ The effects of four-phonon processes, quantum statistics, and different temperatures on the mass ratio dependence of thermal conductivity are intriguing directions for future efforts.

\section{ACKNOWLEDGMENTS}

We thank K. D. Parrish and J. A. Malen (Carnegie Mellon University) for their helpful discussions. This work was supported in part by NSF Grant No. 1133394.

${ }^{1}$ J. M. Ziman, Electrons and Phonons (Oxford University Press, Clarendon, Oxford, 1960).

${ }^{2}$ G. Leibfried and E. Schlomann, Math. Phys. Klasse 4, 71 (1954).

${ }^{3}$ C. L. Julian, Phys. Rev. 137, A128 (1965).

${ }^{4}$ D. P. Spitzer, J. Phys. Chem. Solids 31, 19 (1970).

${ }^{5}$ A. D. McConnell and K. E. Goodson, Annu. Rev. Heat Transfer 14, 129 (2005).

${ }^{6}$ K. Biswas, J. He, I. D. Blum, C.-I. Wu, T. P. Hogan, D. N. Seidman, V. P. Dravid, and M. G. Kanatzidis, Nature 489, 414 (2012).

${ }^{7}$ A. Jain, Y.-J. Yu, and A. J. H. McGaughey, Phys. Rev. B 87, 195301 (2013).

${ }^{8}$ M. Dresselhaus, G. Dresselhaus, and A. Jorio, Annu. Rev. Mater. Res. 34, 247 (2004).

${ }^{9}$ A. J. Minnich, M. S. Dresselhaus, F. Ren, and G. Chen, Energy Environ. Sci. 2, 466 (2009).

${ }^{10}$ M. Zebarjadi, K. Esfarjani, M. S. Dresselhaus, Z. F. Ren, and G. Chen, Energy Environ. Sci. 5, 5147 (2012).

${ }^{11}$ G. A. Slack, J. Phys. Chem. Solids 34, 321 (1973).

${ }^{12}$ L. Lindsay, D. A. Broido, and T. L. Reinecke, Phys. Rev. Lett. 111, 025901 (2013).

${ }^{13}$ D. A. Broido, L. Lindsay, and T. L. Reinecke, Phys. Rev. B 88, 214303 (2013).

${ }^{14}$ L. Wei, P. K. Kuo, R. L. Thomas, T. R. Anthony, and W. F. Banholzer, Phys. Rev. Lett. 70, 3764 (1993).

${ }^{15}$ G. A. Slack, Phys. Rev. 127, 694 (1962).

${ }^{16}$ E. F. Steigmeier and I. Kudman, Phys. Rev. 141, 767 (1966).

${ }^{17}$ A. J. H. McGaughey, M. I. Hussein, E. S. Landry, M. Kaviany, and G. M. Hulbert, Phys. Rev. B 74, 104304 (2006).

${ }^{18}$ F. H. Stillinger and T. A. Weber, Phys. Rev. B 31, 5262 (1985).

${ }^{19}$ J. A. Reissland, The Physics of Phonons (John Wiley and Sons Ltd, 1973).

${ }^{20}$ L. Lindsay, D. A. Broido, and T. L. Reinecke, Phys. Rev. B 87, 165201 (2013).

${ }^{21}$ J. E. Turney, E. S. Landry, A. J. H. McGaughey, and C. H. Amon, Phys. Rev. B 79, 064301 (2009).

${ }^{22}$ D. C. Wallace, Thermodynamics of Crystals (Cambridge University Press, Cambridge, UK, 1972).

${ }^{23}$ M. T. Dove, Introduction to Lattice Dynamics (Cambridge University Press, Cambridge, 1993).

${ }^{24}$ For the mass ratio of 0.25 , where the thermal conductivity is maximum, the thermal conductivities calculated using the RTA and an iterative solution of the $\mathrm{BTE}^{26,28}$ for $N_{o}=44$ are 958 and $1288 \mathrm{~W} / \mathrm{m}-\mathrm{K}$. To save computational effort, the RTA solution, which is up to five times faster than 
the iterative solution of BTE, is employed. We believe that our conclusions will remain unchanged.

${ }^{25}$ K. Esfarjani, G. Chen, and H. T. Stokes, Phys. Rev. B 84, 085204 (2011).

${ }^{26}$ L. Lindsay, D. A. Broido, and T. L. Reinecke, Phys. Rev. Lett. 109, 095901 (2012).

${ }^{27}$ S. Mukhopadhyay and D. A. Stewart, Phys. Rev. Lett. 113, 025901 (2014).

${ }^{28}$ L. Lindsay, D. A. Broido, and T. L. Reinecke, Phys. Rev. B 88, 144306 (2013).

${ }^{29}$ The Debye temperature is calculated using $\theta_{\mathrm{D}}=\frac{\hbar v_{\mathrm{D}}}{k_{\mathrm{B}}}\left(\frac{6 \pi^{2} N_{c}}{V}\right)^{1 / 3},{ }^{1}$ where $v_{\mathrm{D}}$ is the Debye velocity and $N_{c} / V$ is the number density of atoms in the crystal. The Debye velocity is obtained as $3 / v_{\mathrm{D}}^{3}=1 / v_{L A}^{3}+2 / v_{T A}^{3}$, where $\mathrm{v}_{\mathrm{LA}}$ and $\mathrm{v}_{\mathrm{TA}}$ are the longitudinal and transverse acoustic sound speeds in the [100] direction.
${ }^{30}$ The parameter group that appears in the Slack scaling relation is $\bar{m} a \theta_{\mathrm{D}}^{3} /\left(T \gamma^{2}\right)$, where $\gamma$ is the mode-averaged Grüneisen parameter. Because of the difficulty in specifying the mode-averaged Grüneisen parameter, however, $\bar{m} a \theta_{D}^{3} / T$ is generally used to scale thermal conductivity. ${ }^{11}$

${ }^{31}$ P. Giannozzi, S. Baroni, N. Bonini, M. Calandra, R. Car, C. Cavazzoni, D. Ceresoli, G. L. Chiarotti, M. Cococcioni, I. Dabo et al., J. Phys.: Condens. Matter 21, 395502 (2009).

${ }^{32}$ We used an electronic wave-vector grid of $8 \times 8 \times 8$ and a plane-wave energy cutoff of 80 Ryd for all materials. The phonon dispersion is initially calculated on a phonon wave-vector grid of $10 \times 10 \times 10$ using Quantum Espresso and is later interpolated to a phonon wave-vector grid of $18 \times 18 \times 18$. 